\title{
IOM suggests ways to share clinical trial data
}

I $\mathrm{n}$ a bid to address long-standing concerns about accessing clinical trial data, the US Institute of Medicine (IOM) has released a 249-page report outlining strategies for sharing this information responsibly.

"We think that the question today is not whether you share clinical trial data, but instead, what types of data do you share, when do you share and how do you share it?" said Dr. Victor Dzau, president of the Institute of Medicine at a Jan. 14 public briefing on the report.

The report, Sharing Clinical Trial Data: Maximizing Benefits, Minimizing Rights, will have a large impact on researchers globally, says David Moher, a senior scientist with the Ottawa Methods Centre, Clinical Epidemiology Program, Ottawa Health Research Institute. "It would be very nice if ethics committees in Canada would get on board and support this. It will help enormously with issues of downstream research such as doing systematic reviews, meta-analysis and trying to replicate research."

The report calls for widening access to clinical trial data. Currently, most of these data are not posted in a timely manner, much less in an accessible form that could be used by other researchers.

"Perhaps a third of clinical trials do not have the results published for years after the completion of the study," said Bernard Lo, chair of the 13-member IOM Committee on Strategies for Responsible Sharing of Clinical Data. This means that the contributions of participants and clinical trial staff as well as the funding of these trials are not made public. But sharing this information raises questions about who owns the data, how they are going to be used and how to protect the privacy of trial participants.

These are issues that Moher says must be addressed by appropriate policies and procedures before sharing can occur. The IOM report looks, in part, at establishing this framework, although it has no way of enforcing it. Sponsored by 23 public- and private-sector organi-

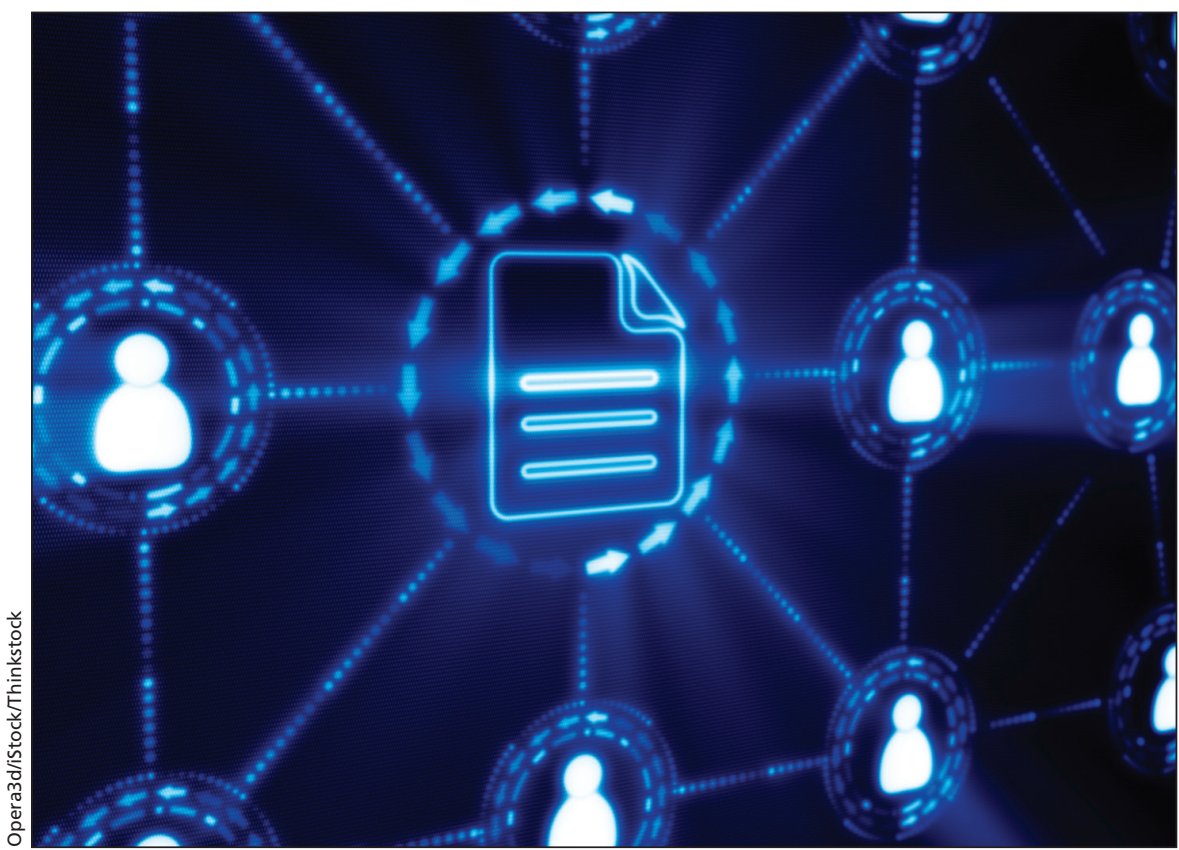

A change in culture is needed to allow timely, transparent and complete sharing of clinical trial data.

zations, the report responds to a 2012 IOM workshop into sharing clinical trial data. It documents four recommendations to improve data sharing.

\section{Making sharing the norm}

The IOM recommends that stakeholders foster a culture in which data sharing is the expected norm. This includes steps that sponsors, investigators, journals, universities and disease advocacy organizations should take to mitigate barriers to sharing data.

For example, "funders and sponsors should require data sharing of the recipients of funding for clinical trials," said Lo. And medical journals should get promises from authors "to share the analytic datasets that support the results of the trial."

The Canadian Medical Association Journal (CMAJ) already requires authors of randomized control trials to submit data-sharing statements with manuscripts. These statements are compulsory, but authors can choose whether to share data and with whom, says Editor-in-Chief Dr. John Fletcher.
However, if journals require that data be shared, as stipulated by the IOM report, it can get more complicated. "Putting together a study — designing it, recruiting patients and recording data is a big undertaking; it's a lot of work. Analyzing it is not so much [work]," says Fletcher. "If you require authors to give away the crown jewels of their study, they've got a lot to lose. So there is a question of how much data [to release] and timing. Those are two crucial details that need to be worked out before we require authors to share their data."

These are concerns that the IOM seeks to address in its timeline of when and what data to release.

\section{Releasing data}

Timely release of data allows for greater scrutiny and discussion surrounding evidence, but is a major concern for sponsors who want to protect intellectual property. The IOM's second recommendation outlines a timeline for sponsors and investigators to ensure optimal release of data during the clinical trial life cycle: 
1. Metadata, including a data sharing plan, should be shared at trial registration.

2. Summary level results and lay summaries should be shared 12 months after study completion.

3. Post-publication data package should be shared within six months of publication.

4. If the regulatory agency does not approve the product, the full data package should be shared 18 months after study completion.

5. If regulatory agency approves the product, a post-regulatory data package should be shared 18 months after product abandonment or 30 days after regulatory approval.

Lay summaries are an important new component that will fulfill the need to disclose information to clinical trial participants, said Lo. "We heard very eloquent testimony from clinical trial participants that they enrolled in a trial and never found out what the investigators found, and we think they need to know what their contributions led to in terms of scientific knowledge."

\section{Ensuring transparency}

The report's third recommendation aims to ensure that data sharing happens fairly. It encourages researchers, sponsors or organizations that hold clinical trial data to implement data-use agreements with other researchers who wish to use the data. In addition, the IOM recommends creating an independent review panel (including public members) that will determine who is able to access the clinical trial data, thereby making the process more transparent.

This recommendation, Lo said, also deals with preventing conflicts of interest when it comes to requesting clinical trial data. "It should not be the company or the principal investigators alone deciding who gets access and who doesn't. It should be an independent neutral party."

Dr. Elizabeth Asztalos, director of clinical trial services at Sunnybrook Research Institute in Toronto, says a major concern is acknowledging who owns the clinical trial data. "The reality is that we have to be very careful as who is defined as owning the data. It's not just the investigators that own the data. Right now, the framework is not there to share data."

"I don't think anyone has any problems sharing data, but we have to recognize the legalities behind it. I can voluntarily want to share data from a trial that I did, but if we didn't do it under the proper legal framework, then we'd get our hands slapped."

\section{Improving infrastructure}

The fourth recommendation concerns the practical challenges of accumulating data in an organized and accessible way. It urges the creation of a multistakeholder body with global reach and a technological enhancement.

Although websites like clinicaltrials. gov, which has 180000 registered studies worldwide, Lo says a lack of a universal and interoperable database is a major problem. "One company may have a way of storing the data and organizing it that is not compatible with how another company does it. If you want to compare two different trials, that's a real headache."

Although newer investigators are embracing a mindset of data sharing, this report is not the end of discussion, said Dr. Ida Sim, professor of medicine at the University of California, and IOM committee member.

"We do need a culture change. For those of us who have been around a little longer, this is a little bit new and I think it's going to take time to transition. The infrastructure, the workforce training - that starts with culture change - I do believe it is starting, and our report is an attempt to move this along." — Dane Wanniarachige, CMAJ

CMAJ 2015. DOI:10.1503/cmaj.109-4980

\section{Exploring physician compensation}

$\mathrm{W}$ hen interviewing celebrities on the podcast Comedy Bang! Bang!, host Scott Aukerman sometimes starts with a rather direct question: "So, how much do you make?" He does this because the question is obviously inappropriate and makes for an awkward (and funny) start to the show, as the star attempts to steer the conversation in another direction.

Personal income is, of course, a personal matter, and the subject is no less touchy in the medical profession than in any other occupation. Though physicians would likely rather discuss how to improve patient care than how much money they make, doctors are nonetheless working professionals, not volunteers, and caring about compensation is no crime.
"It's perfectly fair that physicians have an interest in their income. Everybody does," says Jeremiah Hurley, the chair of economics at McMaster University and a member of the Centre for Health Economics and Policy Analysis.

In most societies, doctors are held in high regard and, in general, are fairly well compensated for their work. In a universal health care system, such as Canada's, physician compensation is a major health care expenditure. How physicians are paid, and how much, can also affect clinical care.

"If you put the incentives in the right place, you can significantly change the way medical practice goes," says Dr. David Attwell, a family physician in Victoria and president of the statutory negotiating committee for Doctors of BC.
$C M A J$ will be exploring physician compensation in a series of articles, touching on issues such as:

Government-physician negotiations: What leverage does each side possess in the secretive and often contentious contract negotiations between provincial governments and physician associations? Do the nature of the negotiations change during times of fiscal restraint? Are there unique elements in this process compared to negotiations between government and other professions? Are governments becoming more involved in influencing individual fees for services rather than only negotiating an overall amount? Is this an appropriate role? 